\title{
A microfluidic based differential plasmon resonance sensor
}

\author{
Melih Okan, Osman Balci, Coskun Kocabas* \\ Bilkent University, Department of Physics, Ankara, Turkey
}

\section{A R T I C L E I N F O}

\section{Article history:}

Received 21 March 2011

Accepted 17 August 2011

Available online 30 August 2011

\section{Keywords:}

Surface plasmon resonance sensor

Phase sensitive detection

Lab-on-a-chip systems

Microfluidic devices

Optical sensors

\begin{abstract}
A B S T R A C T
A new type of differential surface plasmon(SPR) sensor integrated with a microfluidic system is presented. The working principle of the microfluidic device is based on hydrodynamic modulation of two laminar streams inside a microchannel to provide periodic changes of the environment on the SPR sensor. The modulated reflectance is then demodulated using a lock-in amplifier. The presented sensor provides sensitivities of index of refraction about $4 \times 10^{-8}$ RIU together with a 4 orders of magnitude dynamic range. This method demonstrates a sensitive detection scheme which could be used for label-free detection.
\end{abstract} (c) 2011 Elsevier B.V. All rights reserved.

\section{Introduction}

Phase sensitive detection is a remarkably simple technique for recovering weak signals buried in a noisy background. This technique is based on modulation of a signal of interest at a particular frequency by an external parameter and detecting the modulated signal with a phase-sensitive detector (i.e., a lock-in amplifier) [1]. A lock-in amplifier (LIA) amplifies the signal at the frequency of modulation and rejects the uncorrelated signal from noise at other frequencies. Basically LIA demodulates the signal of interest at a particular frequency. Signals with a signal to noise ratio as low as $-100 \mathrm{~dB}$ can be recovered. Noncontact atomic force microscopy is a good example of this sensitive detection method. The method of phase sensitive detection has been combined with many different modulation techniques such as temperature modulation [2], wavelength modulation [3] and spatial modulation [4]. For example light scattering from a single nanoparticle [4] or a carbon nanotube [5] can be detected by modulation of the position of the particle and phase sensitive detection.

In this work we implement the phase sensitive detection technique to microfluidic systems. The idea is based on the periodic modulation of liquid media flowing with a low Reynolds number inside a microfluidic channel. At low Reynolds number regime, the flow inside microfluidic channels is laminar. Taking the advantage of laminar flow, we generate a rapid periodic hydrodynamic modulation of two streams inside the channel without any turbulent mixing. Here one of the laminar streams provide a reference signal

\footnotetext{
* Corresponding author. Tel.: +90 312290 1965; fax: +90 3122664579.

E-mail address: ckocabas@fen.bilkent.edu.tr (C. Kocabas).
}

and the difference between the streams is detected as a differential signal. Similar concepts of hydrodynamic modulations have been applied to electrochemical systems to reduce the background electrochemical currents [6] and to study the frequency response of signaling pathways of cells [7].

Here we implement this hydrodynamic modulation technique with surface plasmon resonance(SPR) sensors. Over the last decade, surface plasmon sensors have attracted much interest owing to their high level of sensitivity and ability of surface specific detection. With a suitable surface chemistry, SPR sensors provide unique means of studying interaction of biomolecules on surfaces. The Kretschmann configuration is most commonly used technique to excite surface plasmon on a flat metal surface. The phase matching condition can be achieved at the resonance angle which is written as

$k_{s p}=k_{0} n_{\text {glass }} \sin \left(\theta_{r}\right)$

where $k_{s p}$ and $k_{0}$ are the wavevector of surface plasmons and excitation photon and $\theta_{r}$ is the resonance angle. The resonance angle depends on the wavevector of the surface plasmon. The dielectric constant of the medium on the metal layer determines the wavevector of the SP. The general sensing mechanism of SPR sensors is based on detecting changes in the intensity of the reflected light as the dielectric constant of the medium changes. The sensitivity of SPR sensors is predicted as high as $10^{-7}$ RIU [8], however, random fluctuations because of laser noise, thermal drift and vibrations significantly reduce the minimum detectable signal of the sensor. Furthermore, during the measurements, the gradual change of the background level, likely because of thermal effects, reduces the repeatability of the measurements. These problems foster the development of new techniques to eliminate the random and 


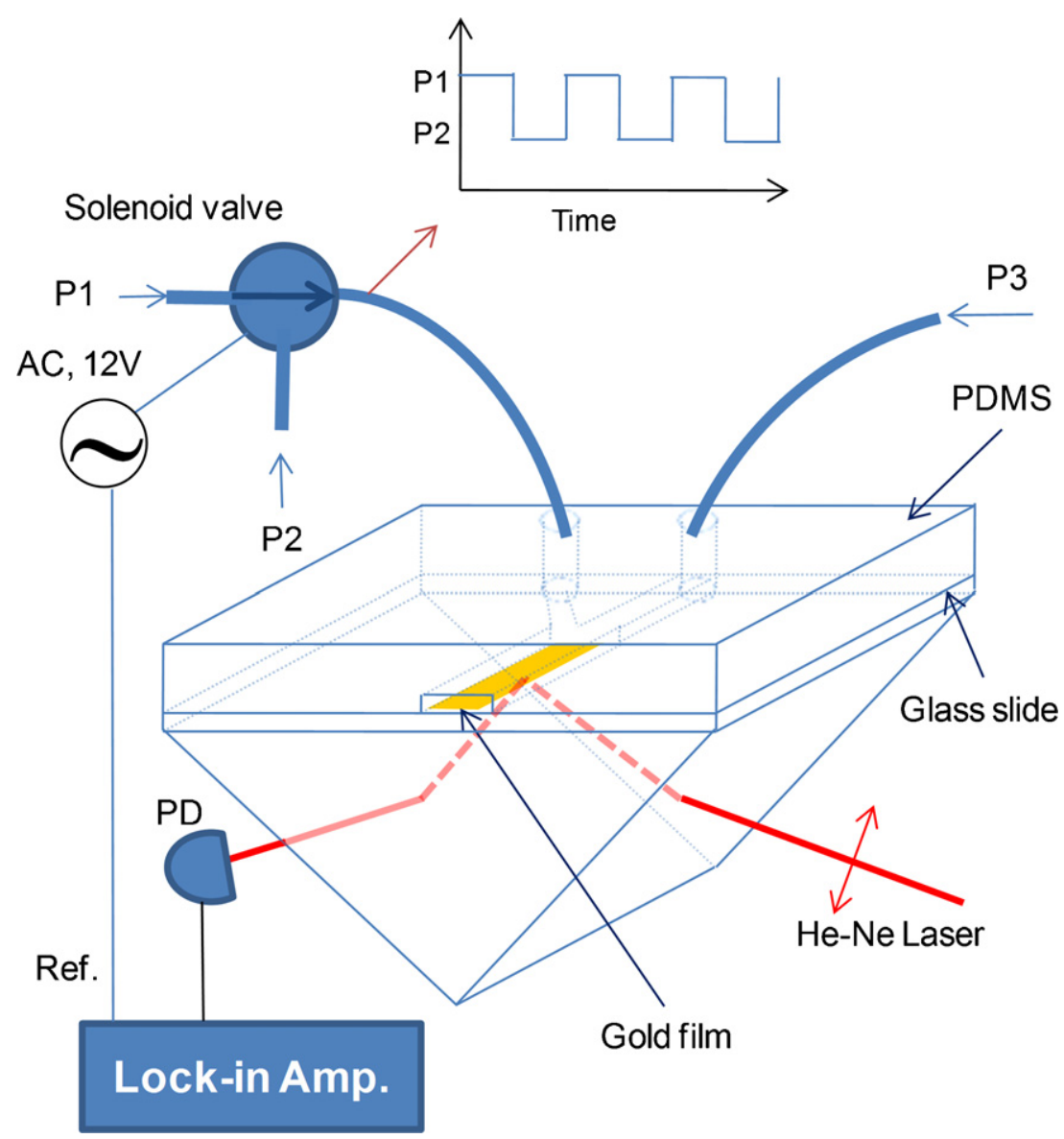

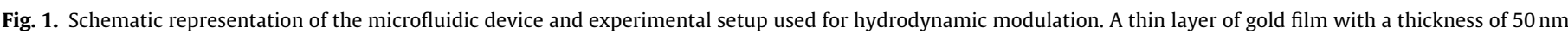

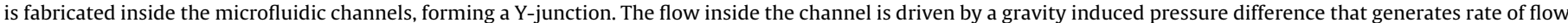

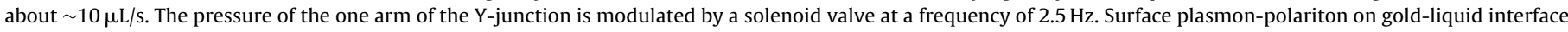

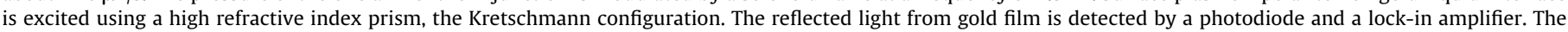
reference signal is used to drive the solenoid valve. The inset graph shows the pressure as a function of time.

systematic errors associated with the experimental setup or the environment. We summaries couple of developed methods to overcome these problems. Wu et al. [9] and Li et al. [10] formed an interferometer which detects the phase change of the reflected light from the metal surface. This method is partially immune of the environmental effects, therefore provides a high level of sensitivity. Zhang et al. [11] introduced a secondary SPR sensor adjacent to the main sensor as a reference. Simultaneously detecting the signal from the reference sensor and the main sensor provides more reliable measurements. More recently, Williams et al. [12] introduced signal-locking SPR sensor which uses a periodic excitation of analyte and a reference SPR sensor with frequency domain signal processing to reduce the uncorrelated signal. These methods and other similar ones [9,13-21] require very tedious alignment for interferometers or a complicated data processing. SPR sensors based on spectroscopy of surface-plasmons integrated with multichannel detectors provide higher sensitivities [22]. Slavík and Homola demonstrated a sensitive SPR sensor based on longrange surface plasmon-polaritons which provides sensitivities of $2.5 \times 10^{-8} \mathrm{RIU}$ [23]. Here, we introduce a simple, yet very sensitive SPR sensor based on a phase sensitive detection scheme using a hydrodynamic modulation inside a microchannel with rates of flow at low Reynolds numbers. The presented method eliminates most of the random fluctuations and background shift during the measurements due to random vibrations, thermal drift and laser noise. The high sensitivity of SPR sensor together with the signal recovery by the phase sensitive detection provide extremely high sensitivities even at very large uncorrelated background noise.

\section{Materials and methods}

Fig. 1 shows the schematic representation of the fabricated microfluidic device. A three-way solenoid valve (LFAA1201418H, The Lee Corporation), derived with a function generator, controls the pressure on the one arm of the Y-junction microfluidic channel. The other arm is kept at a constant pressure. A gravity driven pressure controller is used to control the input pressures. A TM polarized He-Ne laser is used to excite surface plasmons on the gold layer at bottom of the microfluidic channel. The reflected beam is detected with an unbiased photodiode (Newport 818) connected to the lock-in amplifier. The lock-in amplifier (LIA, Stanford Research, SR830) is operated at current amplification mode with a gain of $10^{6}$. The control signal of the valve is used as the reference signal of the LIA. The amplitude and the phase of the differential signal are recorded using a computer and a data-acquisition software.

Fig. 2 shows the fabrication steps of the sensor. The fabrication starts with a standard UV photolithography to pattern the thin layer of gold film (thickness of $50 \mathrm{~nm}$ ) and followed by metallization and lift-off process. The microfluidic channels are fabricated by standard rapid prototyping using the soft lithography technique. A photoresist master (SU-8-50 Micro Chem.) fabricated by UV photolithography is used to mold Polydimethylsiloxane (PDMS) elastomer. PDMS microfluidic channel with a Y-junction 

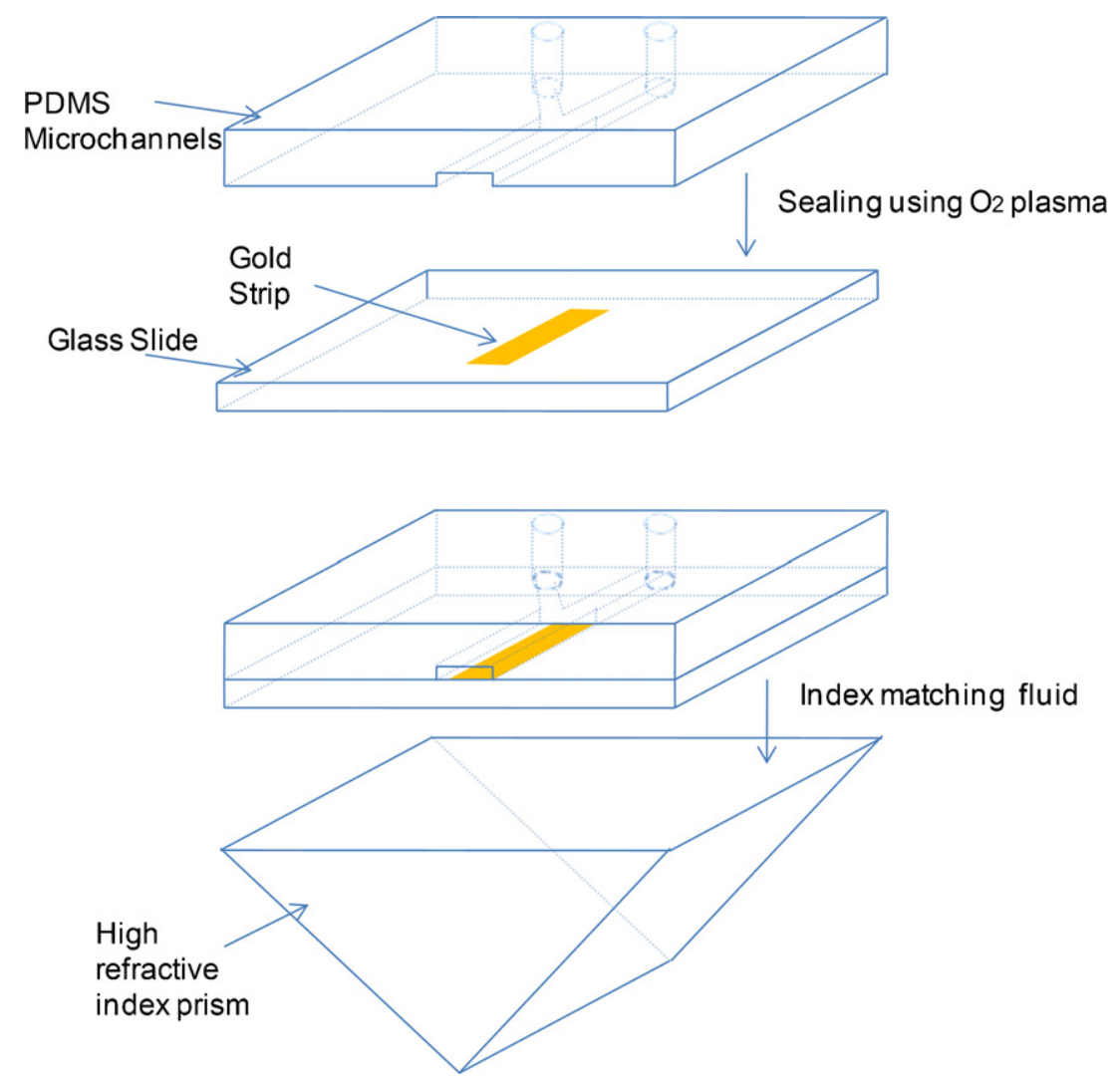

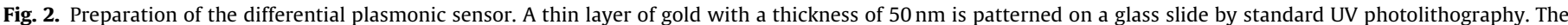

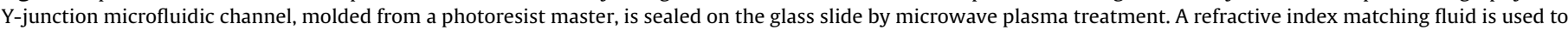
attach the glass slide to the high refractive index prism with refractive index of 1.78 .

geometry is sealed on a glass slide using a $900 \mathrm{~W}$ homemade microwave plasma system. During the sealing process, the gold strip is registered underneath the microfluidic channel. The fabricated microfluidic system is attached on a high refractive index prism (Thorlabs, N-SF11, refractive index of 1.78) using an index matching fluid. The surface plasmon-polaritons on the gold strip is exited by a $5 \mathrm{~mW}$ He-Ne laser with the Kretschmann configuration. A silicon photodiode (Newport 818) connected to the lock-in amplifier is used to detect the intensity of the reflected beam.

\section{Results and discussion}

A three-way solenoid valve is used to control rate of flow of one of the arms. Maximum operation frequency of the solenoid valve is around $200 \mathrm{~Hz}$. Fig. 3a shows the fabricated microfluidic chip which has four SPR sensors. To test the device several issues, such as excitation angle and frequency of modulation, must be considered. First, we generate a laminar flow using two liquids, which are named as liquid $A$ and liquid $B$, with refractive index difference around 0.01 RIU. Here liquid $A$ is DI water $\left(n_{A}=1.33\right)$ and liquid $B$ is $10 \%$ ethyl glycol (EtG, $n_{B}=1.34$ ) in water. Fig. $3 \mathrm{~b}$ and c shows the optical micrographs of the channel filled with liquid $A$ and liquid $B$ for two different pressure levels. The laminar flow boundary between the two streams is seen due to large refractive index difference (0.01 RIU). By switching the pressure of liquid $A$ between two different pressure levels $(\sim 15 \mathrm{kPa}$ and $13 \mathrm{kPa})$ while keeping the pressure of liquid $B$ constant $(\sim 13 \mathrm{kPa})$, we periodically module the index of refraction of the medium on the SPR sensor. As the index of refraction changes, the surface plasmon resonance angle and reflected power changes accordingly. To find the optimum operation point we measure the reflection of the laser beam from the gold surface as a function of incidence angle. Fig. 3d shows the intensity of the laser beam reflected from the gold strip underneath the microfluidic channel filled with liquid $A\left(R_{A}(\theta)\right)$ and liquid $B\left(R_{B}(\theta)\right)$. The reflectivity depends on the incidence angle and the wavelength of the laser. The SPR angle for DI water is $56.76^{\circ}$ and for $10 \%$ EtG is $57.49^{\circ}$. The resonance angle shifts to larger angles as we increase the index of refraction of the liquid by adding ethyl glycol. Fig. 3e shows the change of the intensity of the reflected beam as function of time for three different concentrations of ethyl glycol (10\% (red curve), $2 \%$ (green curve), and $0.4 \%$ (blue curve)). (For interpretation of the references to color in text, the reader is referred to the web version of this article.) For concentrations less than $0.4 \%$, the change in the reflectivity due to the modulation is buried under noise. The modulation of the reflectance provides the differential signal. As the liquids oscillate on top of the gold layer, the SPR angle modulates at the same frequency as well. The difference of the reflectivity for the two cases generates a periodic signal on the detector. The output of the lock-in amplifier provides the amplitude and the phase of the periodic signal between the liquids.

To understand more inside about the working principle of the device, we drive an expression for the differential signal. The reflectivity from the gold surface is a linear combination of $R_{A}(\theta)$ and $R_{B}(\theta)$. The total reflectivity can be written as

$R(\theta)=\frac{x}{w} R_{A}(\theta)+\frac{w-x}{w} R_{B}(\theta)$

where $x$ is the position of the laminar flow boundary which is a periodic function and $w$ is the width of the channel. As the boundary oscillates, the differential signal $(S)$ can be found by taking the 

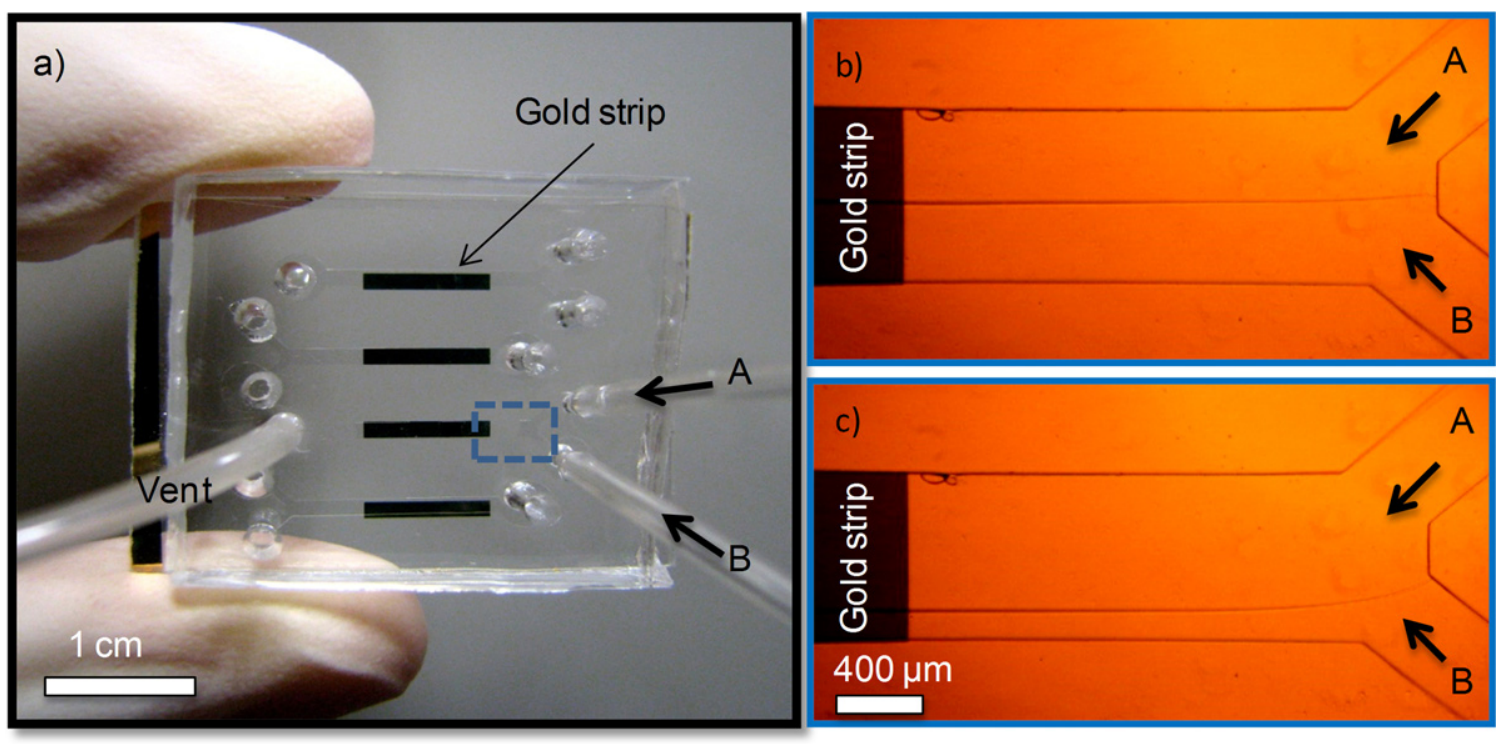

d)

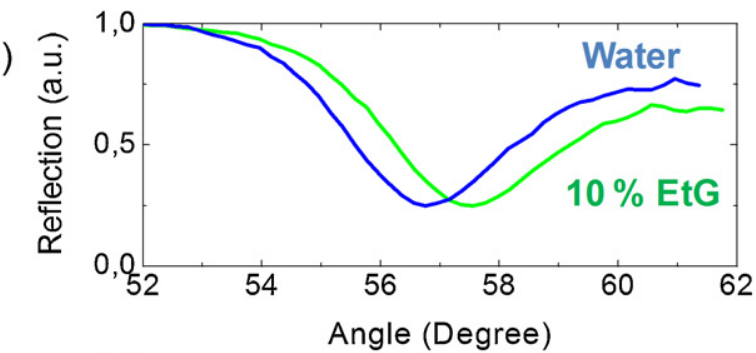

e)

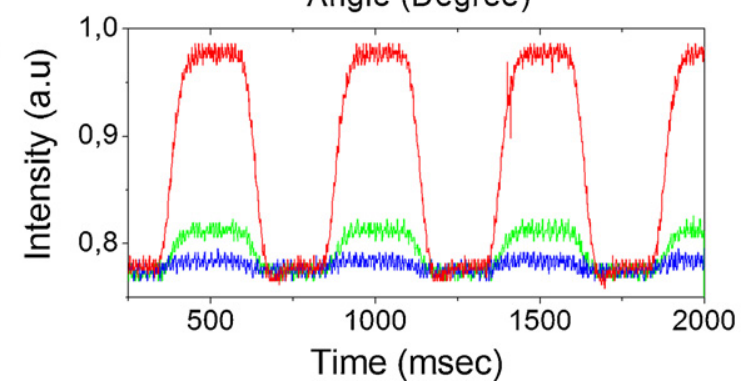

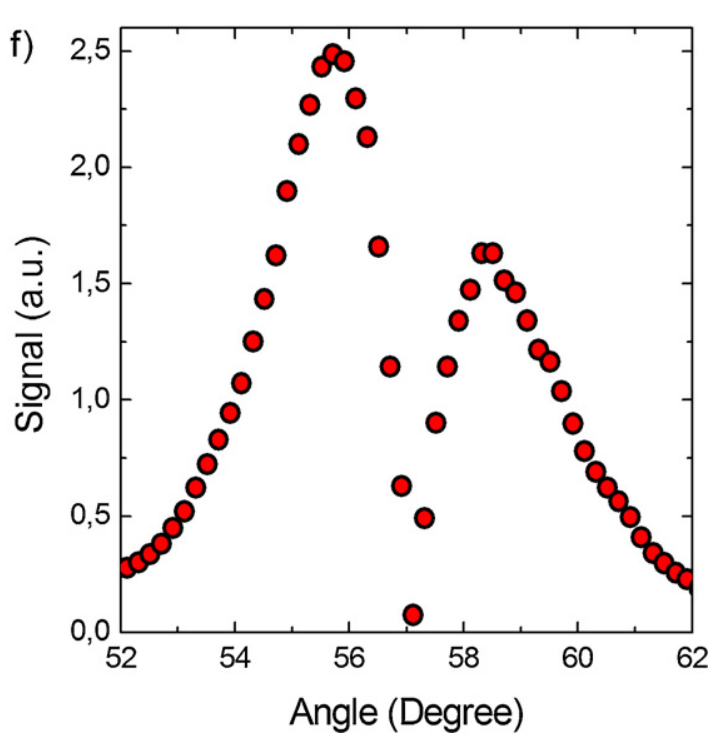

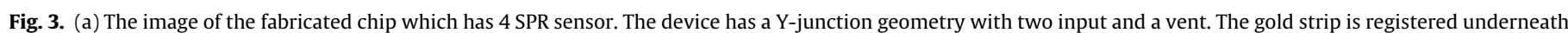

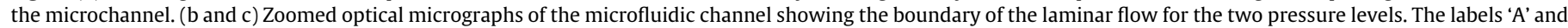

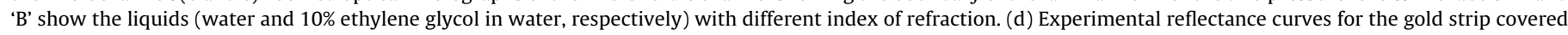

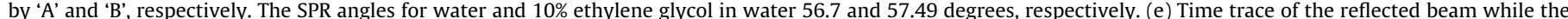

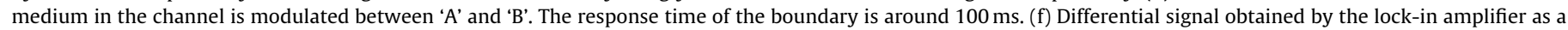
function of the excitation angle. The reference signal of LIA is at $2.5 \mathrm{~Hz}$ and the time constant is $3 \mathrm{~s}$.

difference of the reflection. The differential signal can be written as

$S=\Delta R=\frac{|x|}{w}\left(R_{A}(\theta)-R_{B}(\theta)\right)$

where $|x|$ is the oscillation amplitude of the boundary. For small changes in the index of refraction where the difference between $R_{A}(\theta)$ and $R_{B}(\theta)$ is very small, we can further simplify the equation as

$S=C \frac{|x|}{w} \Delta n \frac{d R(\theta)}{d \theta}$

where $\Delta n$ is the difference in the index of refraction between the liquids, and $C$ is the proportionality constant which defines the change of the resonance angle with respect to the change in index of refraction of the medium. The differential signal is directly proportional with the oscillation amplitude, the difference of index of refraction and the slope of the reflectivity curve. The differential signal obtained for water and $10 \%$ ethyl glycol as a function of the incidence angle is given in Fig. 3f. The signal goes to minima where the reflectivity curves cross and it goes to maxima at an angle which provides the steepest slope in the reflectivity curve.

To understand the mechanism of the hydrodynamic modulation, the frequency dependent measurements are performed. Fig. 4a shows the power spectrum of the signal obtained by changing the internal frequency of the lock-in amplifier. For this measurement the modulation frequency is kept constant at $3 \mathrm{~Hz}$. The time dependent signal shows a nonsinusoidal form which has higher order harmonic components. The intensity of the third harmonic is larger than the second harmonic component because of the square like waveform of the boundary of the two liquids. The frequency dependence of the differential signal is given in Fig. 4a. We obtain the optimum frequency of operation around $2-3 \mathrm{~Hz}$. The optimum frequency of operation depends on the geometry of the channel and the rate of flows. 

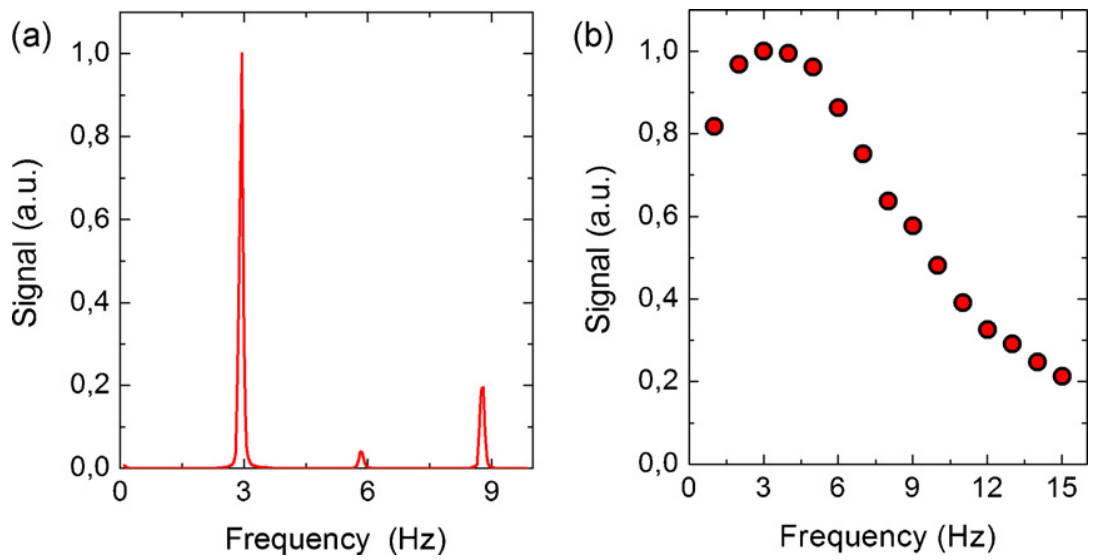

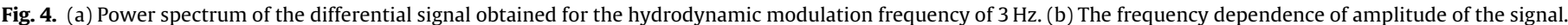

We test the sensitivity of the fabricated sensor by changing the concentration of the ethylene glycol from $5.0 \times 10^{-2} \mathrm{M}$ down to $4.0 \times 10^{-5} \mathrm{M}$. For each concentration we take the power spectrum of the photocurrent and record the differential signal from the spectrum. Fig. 5a shows the power spectrum for various concentrations of EtG. Fig. 5b shows the amplitude of the differential signal as a function of refractive index difference. The signal shows a linear dependence for a very broad range of refractive index change. The fabricated sensor provides very wide dynamic range covering more than 4 orders of magnitude refractive index change. Usually sensitivity and dynamic range of a sensor provide a tradeoff due to the limited bandwidth. The differential SPR sensor simultaneously provides high sensitivity and wide dynamic range. Fig. 5c shows the real time response of the sensor for very low refractive index changes. We add small amount of EtG in water and record the differential signal. A step like response is observed. The calculated change of refractive index for each step is given on the curve. The stability of the background level is also important for reliable measurements. Another advantage of the presented sensor is that background level is constant over a long period of time. The inset at Fig. $5 c$ shows the time trace of the signal level for 1.5 -h time scale. The standard deviation of the signal is around $10 \mathrm{pA}$ which provides a refractive index change of $7 \times 10^{-8}$ RIU. The origin of this extreme stability is that, the two laminar streams provide a self reference detection scheme for thermal drifts. Therefore the background level shows extreme stability over long time scales. The response of the device for low
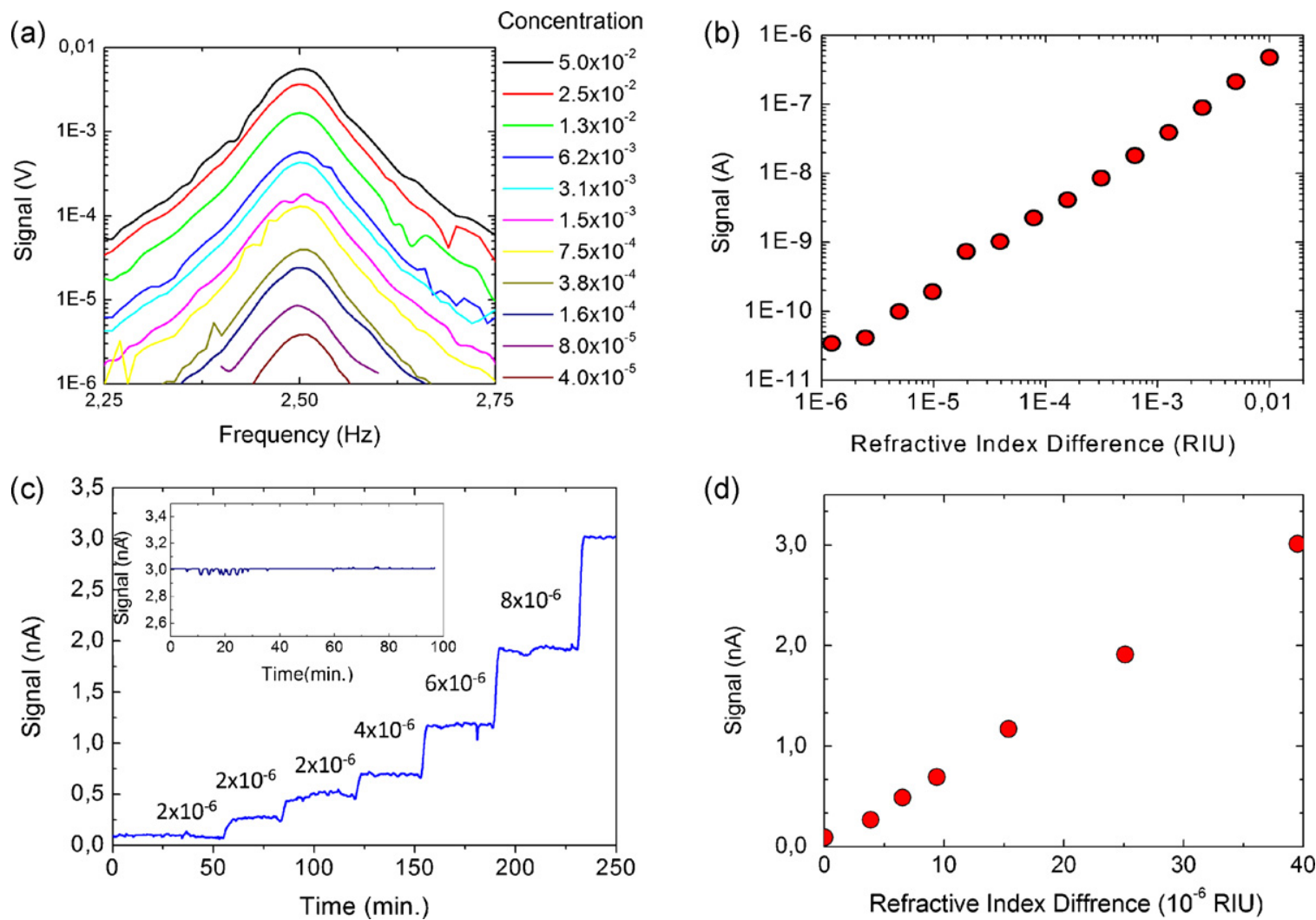

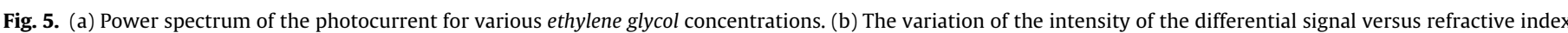

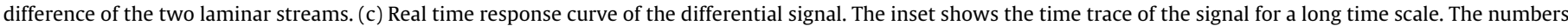

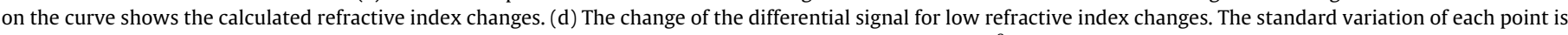
less than the size of the scattered plot. The minimum detectable refractive index difference is around $7 \times 10^{-8} \mathrm{RIU}$ 

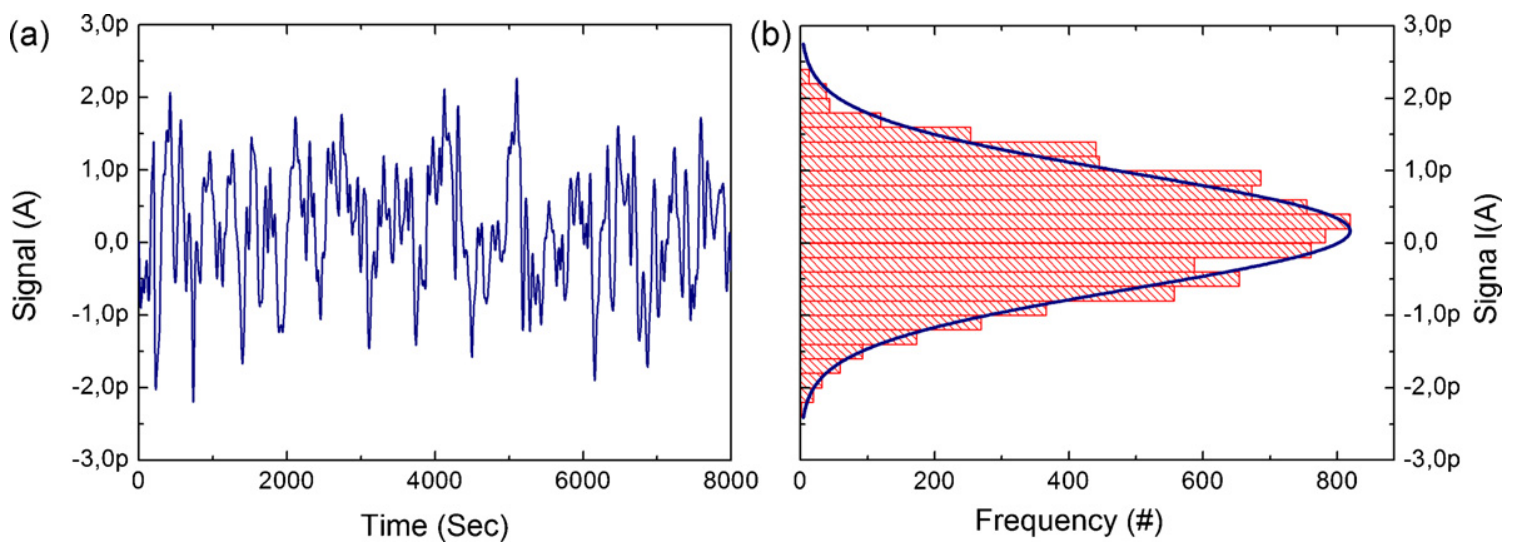

Fig. 6. (a) Variation of the differential signal as a function of time. (b) Histogram of the differential signal.

refractive index changes is given in Fig. $5 d$. The error in the data points is less than the size of the scattered plot.

To understand the ultimate sensitivity limit of the device we analyze sources of noise. Fig. 6a shows the noise of the differential signal. The histogram in Fig. $6 \mathrm{~b}$ has a standard variation around $0.3 \mathrm{pA}$ which corresponds $4 \times 10^{-8} \mathrm{RIU}$. The internal noise of the LIA, shot noise of the detector, and mechanical deformation of the PDMS channel are the main source of noise in the setup. The noise level of the sensor is around $20 \mathrm{pA}$. The internal electronic noise of the LIA is around $0.12 \mathrm{pA}$ for $3 \mathrm{~s}$ time constant at $2.5 \mathrm{~Hz}$. Total noise of the detector and laser is around $2.5 \mathrm{pA}$. The limiting noise source seems due to mechanical deformation or correlated scattering due to impurities in the liquid. Since the laser beam is reflected from the glass side of the device, the mechanical deformation of PDMS during the hydrodynamic modulation does not affect the recorded signal too much. We speculate that the minimum detectable signal is limited by impurities or inhomogeneities in the liquids which generate a correlated noise at the modulation frequency.

\section{Conclusion}

As a conclusion, we present a new type of microfluidic device that uses hydrodynamic modulation scheme for a phase sensitive detection. We implement this method to a surface plasmon sensor integrated in a microfluidic device. The fabricated sensor can detect the refractive index difference as small as $4 \times 10^{-8}$ RIU with an extremely stable signal level and more than 4 orders of magnitude dynamic range. The primary conclusion of this study is that by using a hydrodynamic modulation and phase sensitive detection technique, a simple plasmon sensor with intensity interrogation can provide refractive index sensitivities as high as plasmon sensors with spectral interrogation.

\section{Acknowledgements}

This work was supported by the Scientific and Technological Research Council of Turkey (TUBITAK) Grant No. 110T304 and Marie Curie International Reintegration Grant (IRG) Grant No. 256458.

\section{References}

[1] M. Cardona, Modulation Spectroscopy, Academic Press, 1969.

[2] D. Baurecht, I. Porth, U.P. Fringeli, A new method of phase sensitive detection in modulation spectroscopy applied to temperature induced folding and unfolding of RNase A, Vibrational Spectroscopy 30 (1) (2002) 85-92.
[3] D.S. Bomse, A.C. Stanton, J.A. Silver, Frequency modulation and wavelength modulation spectroscopies: comparison of experimental methods using a leadsalt diode laser, Applied Optics 31 (6) (1992) 718-731.

[4] P. Billaud, S. Marhaba, N. Grillet, E. Cottancin, C. Bonnet, J. Lerme, et al., Absolute optical extinction measurements of single nano-objects by spatial modulation spectroscopy using a white lamp, Review of Scientific Instruments 81 (4) (2010).

[5] F. Wang, D.J. Cho, B. Kessler, J. Deslippe, P.J. Schuck, S.G. Louie, et al., Observation of excitons in one-dimensional metallic single-walled carbon nanotubes, Physical Review Letters 99 (November (22)) (2007).

[6] J. Wang, Hydrodynamic modulation voltammetry, Talanta 28 (6) (1981) 369-376.

[7] P. Hersen, M.N. McClean, L. Mahadevan, S. Ramanathan, Signal processing by the HOG MAP kinase pathway, Proceedings of the National Academy of Sciences of the United States of America 105 (May (20)) (2008) 7165-7170.

[8] J. Homola, S.S. Yee, G. Gauglitz, Surface plasmon resonance sensors: review, Sensors and Actuators B: Chemical 54 (1-2) (1999) 3-15.

[9] S.Y. Wu, H.P. Ho, W.C. Law, C.L. Lin, S.K. Kong, Highly sensitive differential phase-sensitive surface plasmon resonance biosensor based on the Mach-Zehnder configuration, Optics Letters 29 (October (20)) (2004) 2378-2380.

[10] Y.-C. Li, Y.-F. Chang, L.-C. Su, C. Chou, Differential-phase surface plasmon resonance biosensor, Analytical Chemistry 80 (14) (2008) 5590-5595.

[11] H.Q. Zhang, S. Boussaad, N.J. Tao, High-performance differential surface plasmon resonance sensor using quadrant cell photodetector, Review of Scientific Instruments 74 (1) (2003) 150-153.

[12] L.D. Williams, T. Ghosh, C.H. Mastrangelo, Low noise detection of biomolecular interactions with signal-locking surface plasmon resonance, Analytical Chemistry 82 (July (14)) (2010) 6025-6031.

[13] H.P. Ho, W.W. Lam, Application of differential phase measurement technique to surface plasmon resonance sensors, Sensors and Actuators B: Chemical 96 (December (3)) (2003) 554-559.

[14] H.P Ho, W.C. Law, S.Y. Wu, X.H. Liu, S.P. Wong, C.L. Lin, et al., Phase-sensitive surface plasmon resonance biosensor using the photoelastic modulation technique, Sensors and Actuators B: Chemical 114 (March (1)) (2006) 80-84.

[15] A.V. Kabashin, S. Patskovsky, A.N. Grigorenko, Phase and amplitude sensitivities in surface plasmon resonance bio and chemical sensing, Optics Express 17 (November (23)) (2009) 21191-21204.

[16] W.C. Law, P. Markowicz, K.T. Yong, I. Roy, A. Baev, S. Patskovsky, et al., Wide dynamic range phase-sensitive surface plasmon resonance biosensor based on measuring the modulation harmonics, Biosensors \& Bioelectronics 23 (December (5)) (2007) 627-632.

[17] Y.C. Li, Y.F. Chang, L.C. Su, C. Chou, Differential-phase surface plasmon resonance biosensor, Analytical Chemistry 80 (July (14)) (2008) 5590-5595.

[18] P.P. Markowicz, W.C. Law, A. Baev, P.N. Prasad, S. Patskovsky, A.V. Kabashin, Phase-sensitive time-modulated surface plasmon resonance polarimetry for wide dynamic range biosensing, Optics Express 15 (February (4)) (2007) 1745-1754.

[19] S. Patskovsky, R. Jacquemart, M. Meunier, G. De Crescenzo, A.V. Kabashin, Phase-sensitive spatially-modulated surface plasmon resonance polarimetry for detection of biomolecular interactions, Sensors and Actuators B: Chemical 133 (August (2)) (2008) 628-631.

[20] S. Patskovsky, M. Meunier, P.N. Prasad, A.V. Kabashin, Self-noise-filtering phase-sensitive surface plasmon resonance biosensing, Optics Express 18 (July (14)) (2010) 14353-14358

[21] W. Yuan, H.P. Ho, C.L. Wong, S.K. Kong, C.L. Lin, Surface plasmon resonance biosensor incorporated in a Michelson interferometer with enhanced sensitivity, IEEE Sensors Journal 7 (January-February (1-2)) (2007) 70-73. 
[22] J. Homola, Surface plasmon resonance sensors for detection of chemical and biological species, Chemical Reviews 108 (2) (2008) 462-493.

[23] R. Slavík, J. Homola, Ultrahigh resolution long range surface plasmon-based sensor, Sensors and Actuators B: Chemical 123 (1) (2007) 10-12.

\section{Biographies}

Melih Okan is currently a senior undergraduate student at Bilkent University, Department of Physics.
Osman Balci received his BS degree from Bilkent University, Department of Physics in 2009. He is currently a Ph.D. student at Bilkent University, Department of Physics.

Coskun Kocabas received his BS degree in 2001 from Middle East Technical University, Department of Physics. He received his PhD degree from University of Illinois at Urbana Champaign, Department of Physics in 2007. Between 2007 and 2009 he worked as a postdoctoral researcher at Harvard University department of Chemistry and Chemical Biology, Prof. George Whitesides' group. He is currently an assistant professor at Bilkent University, Department of Physics. His core research interests include dynamic microfluidic systems and sensors. 